\title{
Relación entre calidad de vida relacionada con la salud oral, pérdida dental y prótesis removible en adultos mayores de 50 años derechohabientes del IMSS
}

\section{The relationship between oral health- related to quality of life, tooth loss and removable prostheses in IMSS beneficiaries adults over the age of 50}

Bellamy Ortiz Cl*, Moreno Altamirano A**

\section{RESUMEN}

Objetivo: Identificar la relación que hay entre la calidad de vida relacionada con la salud oral (CVRSO) en pacientes con pérdida dental y uso de prótesis removible.

Material y métodos: Estudio transversal en 533 adultos mayores de 50 años de la Unidad Médica Familiar 20 -IMSS-. Se aplicó un cuestionario que incluyó el OHIP-14 (escala que mide la CVRSO) y medición clínica de la pérdida dental y las prótesis removibles.

Resultados: La prevalencia de edentulismo total fue de 9,9\% y de uso de prótesis $35 \%$, de éstas $66 \%$ tenían mala calidad. La media del OHIP-14 fue de 9,83. Se clasificó a los sujetos en siete grupos siguiendo dos criterios: pérdida dental (menos de 20 dientes) y uso de prótesis removible. En la evaluación, el grupo con peor CVRSO fue el grupo que no usaba prótesis removible pero sí requería al tener menos de 20 dientes.

Discusión: El uso de prótesis removible, cuando las personas tienen menos de 20 dientes, mejora la CVRSO aun siendo éstas de mala calidad. Si se tienen 20 dientes o más puede ser una opción no usar prótesis removible para tener una buena calidad de vida relacionada con la salud oral, pero si se tiene menos de 20 dientes sí es necesario.

Palabras clave: Salud bucal, calidad de vida, pérdida de diente, prótesis dental.

\section{SUMMARY}

Objective: To identify the relation between oral health and quality of life (OHRQoL) in patients with tooth loss and prosthesis.

Material and methods: Cross-sectional study in 533 patients of the Instituto Mexicano del Seguro Social (IMSS), which included OHIP-14 and also clinical measures of the oral conditions, considering tooth loss and prosthetic rehabilitation.

Results: The prevalence of total edentulism was of $9.9 \%$, and the use of removable prosthesis of $35 \%$, from which $66 \%$ were of poor quality. The mean of OHIP was of 9.83. Subjects were classified into seven groups according to two criteria: tooth loss (less than 20 teeth) and the use of removable prosthesis. The group with the worst results regarding OHRQoL was the group that did not use removable prosthesis, but required them due to having less than 20 teeth.

* Unidad de Proyectos Especiales de Investigación Sociomédica. Facultad de Medicina UNAM. México DF. ** Departamento de Salud Pública, Facultad de Medicina UNAM. México DF. 
Discussion: The use of removable prosthesis in people with less than 20 teeth improves the OHRQoL, even though the prosthetics may be of poor quality. It may be an option not to use removable dentures when the amount of teeth is more than 20, but it is necessary when people have less than 20 teeth.

Key words: Oral health, quality of life, tooth loss, dental prosthesis.

Fecha de recepción: 14 de junio de 2013.

Aceptado para publicación: 15 de julio de 2013.

Bellamy Ortiz $\mathrm{Cl}$, Moreno Altamirano A. Relación entre calidad de vida relacionada con la salud oral, pérdida dental y prótesis removible en adultos mayores de 50 años derechohabientes del IMSS. Av Odontoestomatol 2014; 30 (4): 195-203.

\section{INTRODUCCIÓN}

La salud bucal es escasamente reconocida por las personas, la sociedad y los servicios de salud, como parte integral de la salud general (1) Entre las funciones de la boca, principalmente se resalta la masticación, obviando importantes aspectos socioculturales como comer, hablar y sonreír. El impacto que tiene la boca dentro de las dinámicas sociales ha sido menos estudiado en comparación con la investigación clínica. En este sentido, en las últimas décadas se han introducido conceptos subjetivos en el campo odontológico. Uno de éstos es el de calidad de vida relacionada con la salud oral (CVRSO), concepto acotado exclusivamente a la relación que tiene la salud oral en la calidad de vida de las personas.

Una de las principales causas que pueden afectar las condiciones biopsicosociales de las personas y uno de los principales problemas de salud bucal pública es la pérdida dental. Ésta es considerada como una deficiencia, de acuerdo con la Clasificación Internacional de Deficiencias, Discapacidades y Minusvalías (CIDDM) propuesta por la OMS en 1980 y retomada por el modelo de Locker, el cual contiene tres dimensiones: orgánica, individual y social. Desde 1982 la OMS propuso conservar al menos 20 dientes bien distribuidos, argumentando que es posible lograr una adecuada funcionalidad; sin embargo, reitera que para lograr una buena salud bucal debe lucharse por la conservación de todos los dientes (2-4).

A pesar de la disminución del edentulismo en algunos países, éste sigue siendo un problema vigente,
Cooper y cols. estimaron que más de 10 millones de nuevos casos se presentarían en la década actual. Lo anterior, aunado al aumento en la esperanza de vida de la población, incrementa a su vez la necesidad de tratamientos bucodentales $(5,6)$.

Desde una mirada clínica, un diente perdido debe ser reemplazado por una prótesis; para esto existen dos opciones: fija o removible. La fija es en algunos casos (y dependiendo de algunas características clínicas que deben cumplirse) un tratamiento de primera elección, el cual tiene algunas ventajas dado que al no ser móvil facilita la adaptación y la estética (7). Pero desafortunadamente su costo es considerablemente más elevado y no es viable como tratamiento generalizado tomando en cuenta las condiciones socioeconómicas de un país como México porque, además, ninguno de los tipos de rehabilitación protésica está incluida como parte de los servicios de salud pública bucal.

Por otro lado, el uso de prótesis removible expone otros problemas. Distintas investigaciones evidencian la controversia acerca de si es mejor o no corregir la pérdida con prótesis removible debido a que existe una alta prevalencia de errores en su elaboración, siendo los principales problemas la estabilidad y la retención. En la Encuesta Nacional de Salud y Nutrición (NHANES III), en Estados Unidos, la prevalencia reportada de prótesis con al menos un defecto fue de $65 \%$ (8). Estos errores pueden producir desajuste de la prótesis, lesiones irritativas en tejidos, reabsorción del hueso alveolar y otras dificultades que producen incomodidad para realizar adecuadamente funciones como comer, hablar y sonreír (9). 
A partir de estos elementos, algunas posturas apuntan que las personas que se rehabilitan con prótesis inadecuadas tienen peor calidad de vida que aquellas que no lo hacen, por lo cual se sugiere que es mejor no utilizar prótesis removible así sea la única opción al alcance. En contraposición, otros autores, entre ellos Inoue y cols., concluyen que la calidad de las prótesis removibles influye mínimamente en la calidad de vida, por lo que su utilización, al ser un tratamiento no tan costoso e invasivo como los implantes, continuará utilizándose ampliamente (10).

La CVRSO también puede verse modificada por factores como edad, nivel socioeconómico y escolaridad, los cuales se encuentran asociados con aumento de pérdida dental. Manifestándose de manera diferente en la percepción, satisfacción del tratamiento, autoestima e imagen corporal (11). La edad es importante por el daño acumulativo que se presenta conforme ésta avanza, por lo que existe una mayor prevalencia de pérdida dental. El nivel socioeconómico y la escolaridad influyen en la utilización o no de rehabilitación protésica por la accesibilidad a los servicios dentales y la calidad de las prótesis que puedan obtener (12).

Por lo descrito anteriormente, el objetivo del estudio intenta responder a la pregunta de ¿Cuál es la relación que existe entre la CVRSO, la pérdida dental y las prótesis removibles en adultos mayores de 50 años?

\section{MATERIALES Y MÉTODOS}

Estudio transversal realizado en una muestra de 533 adultos mayores de 50 años que acudieron a consulta externa en la Unidad de Medicina Familiar núm. 20 del Instituto Mexicano del Seguro Social (IMSS) durante los meses de abril, mayo y junio del 2010. El proyecto fue aprobado por la comisión de investigación y ética de la Facultad de Medicina de la UNAM. Para el cálculo del tamaño de la muestra se consideró un puntaje de siete (obtenido del estudio piloto) de la escala de calidad de vida relacionada con la salud oral, el Oral Health Impact Profile (OHIP-14) con un nivel de confianza de $95 \%$ y una precisión de 0,02 para obtener un tamaño muestral de 408 sujetos se incrementó un $20 \%$ para disminuir las posi- bles pérdidas evaluando un total de 533 pacientes. Los criterios de inclusión utilizados fueron pacientes que acudieran a la clínica a consulta externa y que accedieran a participar en el estudio. Se les hizo una revisión bucodental y se les dio un diagnóstico de salud oral, sugiriendo un orden en las prioridades de atención. Cuatro odontólogos previamente capacitados y estandarizados aplicaron el cuestionario, que contenía variables sociodemográficas, y una revisión clínica, que constaba del diagnóstico de la calidad de las prótesis tomando en cuenta estabilidad y retención medidas con los criterios de Catovic y cols. (13). Se registraron las condiciones dentales en un odontograma basado en los métodos de la OMS (14). El OHIP-14 es un instrumento específico que mide la calidad de vida relacionada con la salud oral a partir del impacto de las enfermedades orales, desarrollado por Slade y Spencer para proporcionar una medida autorreportada de disfunción, discapacidad y desventaja (15). Los impactos están conceptualizados como efectos adversos. El instrumento tiene siete dimensiones: limitación funcional, dolor físico, discapacidad psicológica, incapacidad física, incapacidad social, incapacidad psicológica y en desventaja. Las respuestas se basan en una escala tipo Lickert con un puntaje de 0 (sin impacto en salud oral) a 56 (peor salud oral). El instrumento está validado en el lenguaje español y también se encuentra validado y adaptado en población mexicana (16-18).

Las variables sociodemográficas registradas fueron: edad, sexo, escolaridad, analfabetismo, escolaridad del jefe de familia, actividad, gasto mensual, número de personas con las que vivía y número de medicamentos que consume. También se identificaron enfermedades como hipertensión y diabetes mellitus tipo 2, ambas autorreportadas. Se hicieron algunas preguntas en relación al uso de prótesis, el número total de prótesis a lo largo de la vida, tiempo de utilización, problemas en su uso, razones de no uso, lugar y costo donde se realizaron.

Se agruparon a los pacientes combinando tres criterios de acuerdo a las condiciones dentales:

A) Pérdida dental.

B) Si requería prótesis, tomando como referencia el parámetro propuesto por la OMS de tener al menos 20 dientes.

C) Uso de prótesis removibles. 
De esta manera, de acuerdo a estas condiciones dentales, los sujetos se clasificaron en siete grupos (Tabla 1).

El análisis de la información se realizó por medio del programa SPSS v19. Se estimaron las prevalencias del uso de prótesis en general y la calidad de éstas. También se determinó la puntuación obtenida en el $\mathrm{OHIP}$, en todas sus dimensiones y se obtuvieron medidas de tendencia central y dispersión en las variables cuantitativas. Posteriormente se realizaron análisis bivariados utilizando pruebas de ANOVA y $\mathrm{X}^{2}$ con las distintas variables para identificar la asociación entre ellas.

\section{RESULTADOS}

La muestra total de pacientes entrevistados fue de 533. En la tabla 2 se muestran las características sociodemográficas de la población estudiada, el promedio de edad fue de 67,4 (DE $\pm 8,3$ ), la mayoría

\begin{tabular}{|l|c|c|c|}
\hline \multicolumn{4}{|c|}{ TABLA 1.- CLASIFICACIÓN DE } \\
CONDICIONES DENTALES SEGÚN \\
CRITERIOS DE PÉRDIDA DENTAL Y USO DE \\
PRÓTESIS REMOVIBLE
\end{tabular}

estaba casado 69,6\%. El 56,5\% fueron mujeres, de las cuales el $80 \%$ se dedicaban al hogar, el $61 \%$ de los hombres eran jubilados. El 38,6\% eran diabéticos y $52,2 \%$ hipertensos.

Se encontró que la prevalencia de uso de prótesis fue de $35 \%$; de éstas $66,1 \%$ tenían mala calidad. Entre las personas que utilizaban prótesis parcial el $66,2 \%$ eran mujeres y entre los que empleaban prótesis total el $34,5 \%$ eran hombres.

Del total de la muestra, el 46,5\% afirmó haber utilizado prótesis alguna vez. Entre los portadores de prótesis el $79 \%$ mencionó utilizar su prótesis dental permanentemente y el $43 \%$ dijo tener problemas con éstas. El $41,4 \%$ había cambiado alguna vez su prótesis, el $55 \%$ había tenido sólo una y $25 \%$ dos. El promedio de tiempo usando prótesis fue de ocho años, con un rango de un mes a 50 años. El $84,9 \%$ se hizo la prótesis con un dentista particular y 55,4\% pagó más de dos mil pesos (U\$ 160) por éstas. El 37,5\% de las personas reportó tener problemas para comer, 38,8\% dijo tardar más tiempo para comer y 32,5\% había dejado de comer algún alimento.

En cuanto a las características dentales, los pacientes tuvieron en promedio 16,9 dientes presentes, de los cuales, 8,6 estaban sanos; 5,5, cariados y 1,9, obturados. La media de dientes perdidos fue de 13,3. La prevalencia de edentulismo fue de 9,9\%.

En este estudio, el promedio del OHIP-14 fue de 9,8 (DE $\pm 10,0)$ con un puntaje máximo de 47 . En todas las dimensiones se obtuvo un rango de 0 a 8 . De las siete dimensiones, la que obtuvo un valor más alto fue la limitación funcional con una media de 2,4; en dolor físico 1,9, seguida de la incapacidad física con 1,9. El puntaje más bajo se encontró en la incapacidad social con 0,6. El grupo con los valores más altos en las siete dimensiones fue el de "No usa prótesis pero sí requiere".

\section{DISCUSIÓN}

Los resultados obtenidos muestran que las condiciones dentales con respecto a la pérdida dental y el uso de prótesis removible influyen en la CVRSO. Dependiendo de las distintas combinaciones en es- 


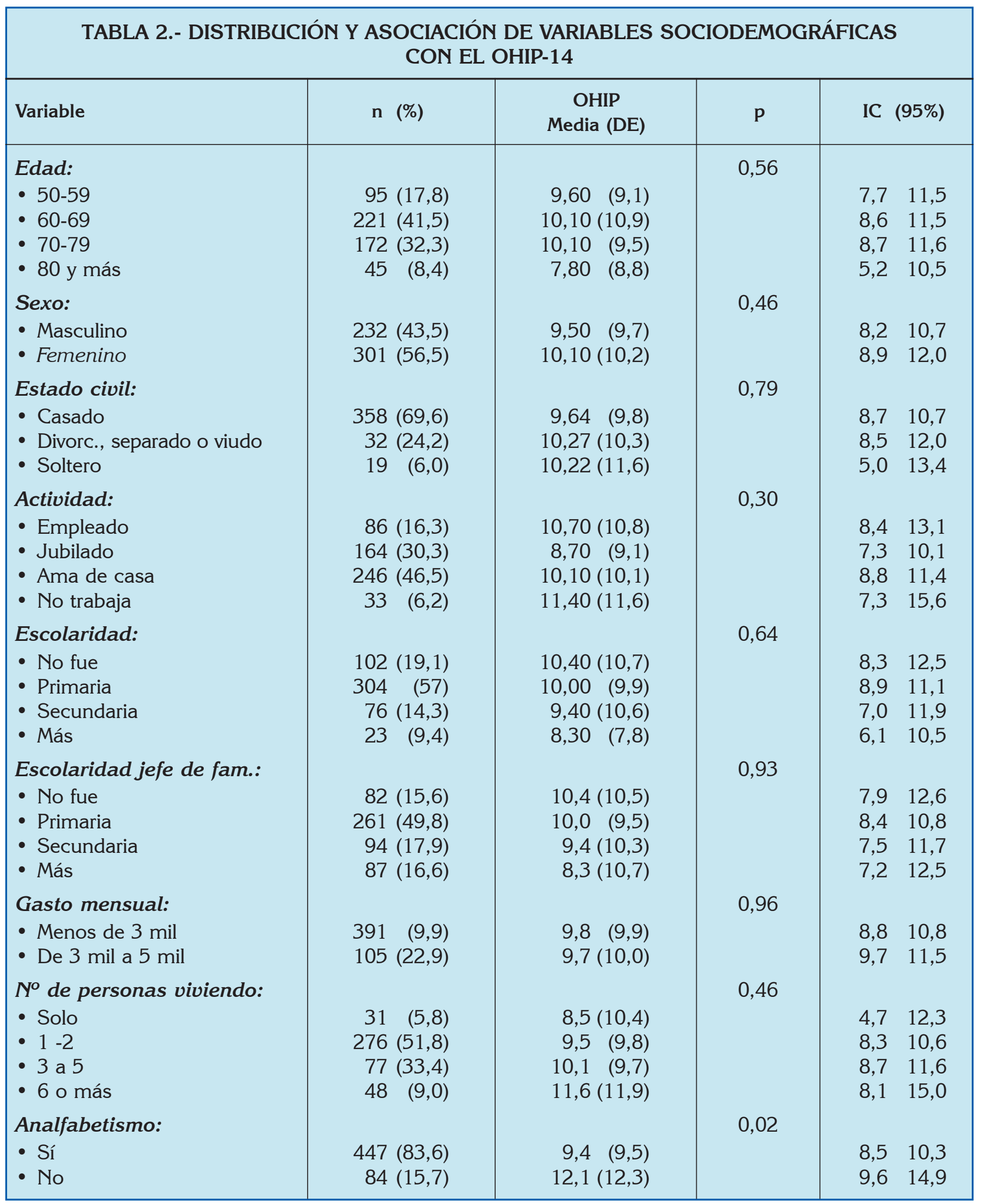


tas dos condiciones la CVRSO puede ser mejor o peor, si se tienen 20 dientes puede ser una opción no utilizar prótesis pero si se tienen menos de 20 dientes sí es necesario. Como se puede apreciar en los resultados los grupos que tuvieron mejor CVRSO de acuerdo a la media obtenida con el instrumento OHIP fueron en primer lugar los que utilizaban prótesis total $(5,3)$, luego los que no tenían pérdida dental $(6,5)$, el siguiente grupo mejor evaluado fue el que no utilizaba prótesis y no la requería al tener 20 dientes $(8,2)$ con una media similar al grupo de los que sí la requerían y sí la usaban $(9,2)$, y al grupo de los edéntulos $(9,0)$. Los grupos peor evaluados fueron los que utilizaban prótesis parcial pero no la requerían porque tenían 20 dientes $(10,7)$ y los que no usaban prótesis pero sí la requerían $(17,2)$ (Tabla 3).

En todos los grupos que utilizaban prótesis con respecto a la calidad de éstas, se encontró un mejor puntaje de CVRSO si eran de buena calidad con una media de 7,4 en comparación con los grupos de portadores de prótesis con mala calidad que tuvieron una media de 9,1. El aumento más notorio fue en el grupo que usaba prótesis parcial pero no la requería con una media de 8,0 si su prótesis era de buena calidad comparado con una media de 16,6 si era de mala calidad, media similar al grupo que no usaba pero sí requería $(17,2)$, ambos fueron los pun-

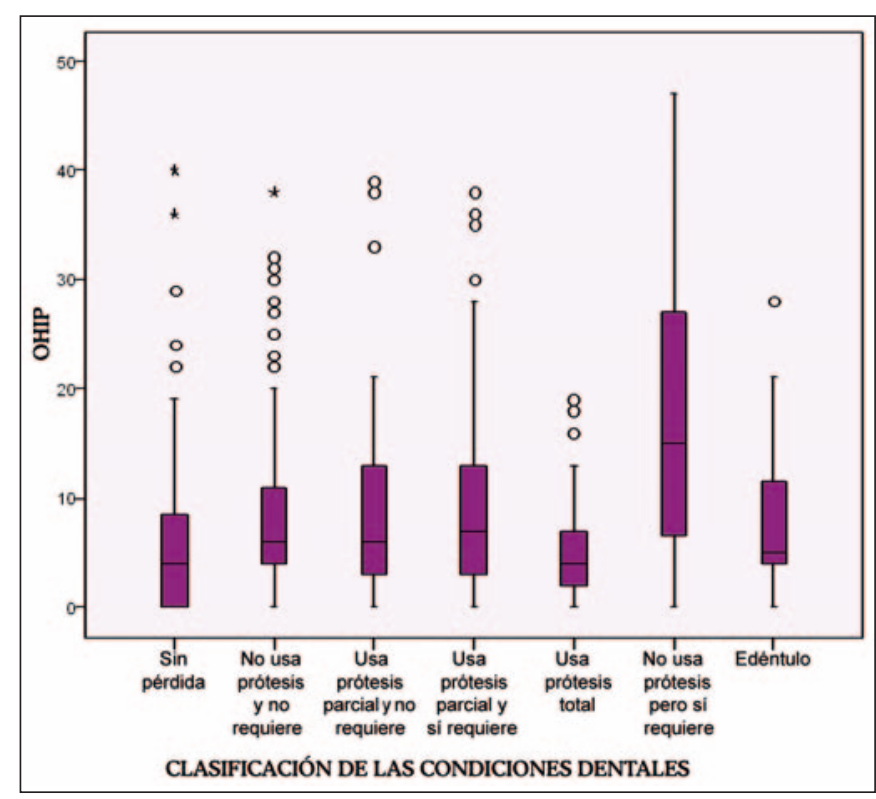

Graf. 1. Medias obtenidas con el instrumento Oral Health Impact Profile (OHIP-14) en la clasificación de condiciones dentales.

tajes más altos, es decir la peor CVRSO que se encontró en los distintos grupos. No obstante, si se tienen menos de 20 dientes, es mejor tener una rehabilitación protésica, aún si ésta tiene calidad deficiente, dado que en las personas rehabilitadas la

TABLA 3.- DISTRIBUCIÓN Y ASOCIACIÓN DEL OHIP-14 CON LAS CONDICIONES DENTALES

\begin{tabular}{|c|c|c|c|c|c|c|c|}
\hline \multirow{2}{*}{ Variable } & \multicolumn{2}{|r|}{$\mathrm{n}$} & \multicolumn{2}{|c|}{ OHIP } & \multicolumn{3}{|c|}{ Significancia* } \\
\hline & Frec & (\%) & Media & $(D E)$ & $\mathrm{p}$ & (IC & $95 \%)$ \\
\hline $\begin{array}{l}\text { Clasificación de condiciones dentales } \\
\text { - Sin pérdida } \\
\text { - No usa prótesis y no requiere } \\
\text { - Usa prótesis parcial y no requiere } \\
\text { - Usa prótesis parcial y sí requiere } \\
\text { - Usa prótesis total } \\
\text { - No usa prótesis pero sí requiere } \\
\text { - Edéntulo }\end{array}$ & $\begin{array}{r}71 \\
165 \\
22 \\
120 \\
41 \\
103 \\
11\end{array}$ & $\begin{array}{r}(13,3) \\
(31,0) \\
(4,1) \\
(22,4) \\
(7,7) \\
(19,3) \\
(2,1)\end{array}$ & $\begin{array}{r}6,5 \\
8,2 \\
10,7 \\
9,2 \\
5,3 \\
17,1 \\
9,0\end{array}$ & $\begin{array}{r}(8,5) \\
(7,6) \\
(11,9) \\
(8,3) \\
(4,9) \\
(13,2) \\
(8,7)\end{array}$ & $<0,001$ & $\begin{array}{r}(4,5 \\
(7,0 \\
(5,5 \\
(7,7 \\
(3,7 \\
(14,5 \\
(3,1\end{array}$ & $\begin{array}{r}8,5) \\
9,4) \\
16,0) \\
10,7) \\
6,8) \\
19,7) \\
14,9)\end{array}$ \\
\hline Total & 533 & $(100,0)$ & 9,8 & $(10,0)$ & & $(9,0$ & $10,7)$ \\
\hline
\end{tabular}


CVRSO mejora (9,8 sí utilizaba prótesis parcial y 5,4 sí utilizaba prótesis total), en comparación con aquellos que tenían menos de 20 dientes pero no estaban rehabilitados $(17,2)$ (Tabla 4$)$.

En general, las dimensiones más afectadas en la CVRSO fueron las de limitación funcional, dolor físico e incapacidad física. Es decir, dificultades en aspectos como masticar, la apariencia, el dolor, la forma de hablar y de sonreír. Las tres dimensiones más afectadas coinciden con las reportadas por otros autores entre ellos Öshayat y cols. (19) que también estudiaron población con pérdida dental y uso de prótesis fija y removible.

Además, se comprueba que la pérdida de dientes continúa siendo uno de los principales problemas de salud pública bucal. El 97\% de la población había

\section{TABLA 4.- MEDIA OBTENIDA CON EL INSTRUMENTO OHIP DE ACUERDO A LA CLASIFICACIÓN DE CONDICIONES DENTALES SEGÚN CALIDAD DE LAS PRÓTESIS REMOVIBLES}

\begin{tabular}{|c|c|c|c|}
\hline \multirow[b]{2}{*}{$\begin{array}{l}\text { Clasificación de } \\
\text { condiciones dentales }\end{array}$} & \multicolumn{2}{|c|}{$\begin{array}{l}\text { Calidad } \\
\text { de la prótesis }\end{array}$} & \multirow[b]{2}{*}{ Total } \\
\hline & $\begin{array}{c}\text { Buena } \\
n=121 \\
(66,1 \%)\end{array}$ & $\begin{array}{c}\text { Mala } \\
n=62 \\
(33,9 \%)\end{array}$ & \\
\hline $\begin{array}{l}\text { - Sin pérdida } \\
\text { - No usa prótesis y no } \\
\text { requiere } \\
\text { - Usa prótesis parcial } \\
\text { y no requiere } \\
\text { - Usa prótesis parcial } \\
\text { y sí requiere } \\
\text { - Usa prótesis total } \\
\text { - No usa prótesis pero } \\
\text { sí requiere } \\
\text { - Edéntulo }\end{array}$ & $\begin{array}{c}8,0 \\
7,8 \\
4,9 \\
- \\
-\end{array}$ & $\begin{array}{c}- \\
- \\
16,6 \\
9,8 \\
5,4 \\
- \\
-\end{array}$ & $\begin{array}{r}6,5 \\
8,2 \\
10,7 \\
9,2 \\
5,3 \\
17,2 \\
9,0\end{array}$ \\
\hline Total & 7,4 & 9,1 & 9,8 \\
\hline
\end{tabular}

perdido uno o más dientes, con una media de 13,3 $(\mathrm{DE} \pm 9,3)$ dientes perdidos. Con respecto al uso de prótesis removible, el 34\% utilizaba una prótesis parcial y 53 individuos eran edéntulos totales, de los cuales $80 \%$ usaba prótesis total.

En relación con la calidad de las prótesis evaluadas según estabilidad y retención, siguiendo los criterios de Catovic y cols., la prevalencia de mala calidad fue del $66 \%$, cifra similar a la reportada por estos autores. Igualmente se encontró que las personas que tenían prótesis de buena calidad, tenían una mejor CVRSO, con un puntaje de 7,4 , y en los que tenían mala calidad, el valor fue 9,1. Cabe resaltar que de los sujetos que usaban prótesis de mala calidad, el $47,6 \%$ dijeron no haberla cambiado porque no tenían problemas con éstas, como afirman Begum y cols, donde establecen que la satisfacción del paciente no se basa únicamente en la calidad técnica de las dentaduras, sino en la adaptación (20). Así, es posible que los adultos mayores tengan un mayor grado de adaptación y aceptación de su propia condición, asociada a la edad y, en consecuencia, a la acumulación de daños bucales como parte del reconocimiento popular de asumir la pérdida dental dentro del proceso natural de envejecimiento, como se observa en la tabla I donde el grupo con la media más baja es el de 80 y más. Igual sucede con los edéntulos portadores de prótesis total, quienes reportan un mejor puntaje en la CVRSO en relación a otros grupos $(5,3)$. Esto coincide con la revisión hecha por Carlsson y cols. donde se establece que entre el $65 \%$ y $90 \%$ de los pacientes edéntulos están satisfechos con sus prótesis removibles (21).

Esto no quiere decir que no se deba tener en cuenta la calidad de las prótesis, siendo éste un parámetro fundamental para un tratamiento ideal. Los resultados muestran una mejoría que concuerda con lo reportado por Allen, quien establece que la mala calidad de las prótesis afecta la CVRSO. Aún así, se puede debatir las propuestas que sugieren no utilizar prótesis removible si no hay garantías en la calidad (22).

Nuestra postura no coincide con este planteamiento, porque según los resultados obtenidos, es peor no rehabilitar cuando se tienen menos de 20 dientes. Tampoco se puede considerar como una opción viable otro tipo de rehabilitación de acuerdo a las 
actuales condiciones sociales y altos costos de otros tratamientos, pues de acuerdo con la Encuesta Nacional de Ocupación y Empleo (ENOE) el 74\% de la población económicamente activa gana entre uno y cinco salarios mínimos (154 a 771 USD). Debido a que la rehabilitación protésica no está incluida dentro de los esquemas de seguridad social en salud, las personas la obtienen en otros espacios, generalmente en la consulta privada; $85 \%$ obtuvieron su prótesis con un dentista particular. Esta consideración es importante si tomamos en cuenta que en la población estudiada el $73,4 \%$ tenía un gasto mensual de menos de tres mil pesos por familia (235 USD). Además 36,8\% de los entrevistados dijo no haber cambiado su prótesis por falta de dinero y $52 \%$ pagó más de dos mil pesos (más de 157 USD) por su prótesis (23).

Por otro lado, los resultados indican que la medición objetiva y subjetiva de la salud oral son entidades conceptuales distintas. No podemos poner en contradicción un constructo subjetivo como lo es el de CVRSO con una medida como la pérdida dental (24). Son medidas complementarias que permiten tener un perspectiva de análisis más compleja donde se integra la percepción del paciente sobre su salud oral y el impacto en su bienestar.

En conclusión, en lo relacionado a la rehabilitación de la pérdida dental, los resultados obtenidos muestran que las prótesis removibles, aunque no sean la mejor elección en algunos casos, siguen siendo una opción viable que mejora el impacto en la CVRSO por sus características de ser más económica que otras opciones, estéticamente aceptable y fácil de limpiar. Sin embargo, hay que considerar algunas cuestiones; una de éstas es la disponibilidad de recursos económicos y la otra el número de dientes presentes ya que, si se tienen 20 dientes o más ,es una opción no usar prótesis removible para tener una buena CVRSO pero si se tiene menos de 20 dientes sí es necesario pero preferentemente con estándares de calidad.

\section{AGRADECIMIENTOS}

Las autoras desean expresar su agradecimiento al Instituto Mexicano del Seguro Social y al personal de la Unidad Familiar No. 20 por su colaboración para poder concluir este proyecto. También deseamos agradecer el financiamiento otorgado por parte del departamento de Salud Pública de la Facultad de Medicina de la UNAM con el cual se realizo el trabajo de campo.

\section{BIBLIOGRAFÍA}

1. Pyle MA. Changing perceptions of oral health and its importance to general health:provider perceptions, public perceptions, policymaker perceptions. Spec Care Dentist 2002;22(1):8-15.

2. World Health Organization (WHO) International Classification of impairments, Disabilities and Handicaps. A manual of classification relating to the consequence of disease. Geneva: WHO; 1980.

3. Locker D. Measuring oral health: a conceptual framework. Community Dent Health 1988;5:318.

4. World Health Organization (WHO) Recent advances in oral helath. WHO Technical Report Series. No.826. WHO, Geneva, 16-7.

5. Cooper L. The current and future tretment of edentulism. Journal of Prosthodontics 2009;18: 116-22.

6. Furuyama C, Takaba M, Inukai M, Mulligan R,Igarashi Y, Baba K. Oral Health related quality of life in patients treated by implant-supported fixed dentures and removable partial dentures. Clin Oral Impl 2011;20:1-5.

7. Gonçalves W, Gláucia G, Aparecida J, Adelino V. Comparing the efficacy of mandibular implantretained overdentures and conventional dentures among elderly edentulous patients satisfaction and quality of life. Gerodontology 2007;24:235-8.

8. Hummel SK, Wilson MA, Marker VA, Nunn ME. Quality of removable partial dentures worn by the adult U.S. population. The Journal of Prosthetic Dentistry 2002;88(1):37-43. 
9. Liedberg B, Stoltze K. The masticatory handicap of wearing removable dentures in elderly men. Gerodontology 2005;22:10-6.

10. Inoue M, John MT,Tsukasaki H, Furuyama C, Baba K. Denture Quality has a minimal effect on health related quality of life in patients with removable dentures. Journal of Oral Rehabilitation 2011;28:818-26.

11. John MT, Koepsell TD, Hujoel P, Miglioretti DL, Le Resche L, Micheeli W. Demographic factors, denture status and oral health-related quality of life. Community Dent Oral Epidemiol 2004;32: 125-32.

12. Kandelman D, Petersen PE,Uleda Hiroshi. Oral health, genral health, and quality of life in older people. Spec Care Dentist 2008;28(6):224-35.

13. Catovic A, Bergman V, Catic A. Qualitative evaluation of elderly home residents' fixed and removable prostheses in relatión to the ADL index. The Journal of Dentistry 2003;31:3-8.

14. World Health organiztion. Ora Health Surveys:basic methods. Geneva: WHO, 1977.

15. Slade G. The oral health impact profile. Measuring oral health and quality of life.Chapel Hill. University of North Carolina. Dental Ecology 1997:93-104.

16. Locker D. The concept of positive health: a review and commentary on its application in oral health research. Community Dental Oral Epidemiology 2006; 34:161-73.

17. López R, Baelum V. Spanish version of the oral health impact profile (OHIP-Sp). BMC Oral Health 2006;6:11.

18. Castrejón R, Borges A, Irigoyen M. Validación de un instrumento para medir el efecto de la salud bucal en la calidad de vida de adultos mayores mexicanos. Rev Panam. Salud Pública 2010;27 (5):321-9.

10. Öshayat E, Gotfredsen K. Oral health-relatedquality-of-life in patients to be treated with fixed or removable partial dental prostheses. Acta Odontol Scand 2012:1-7.

20. Begum S, Damla I, Kulak Y. Satisfaction of the complete denture wearers related to various factors. Archives of Gerodontology and Geriatrics 2009;49:126-9.

21. Carlsson GE, Omar R. The future of complete dentures in oral rehabilitation. A critical review. Journal of Oral Rehabilitation 2010;37:143-56.

22. Allen F. Assesment oral health related quality of life. Health quality of life outcomes. 2003;1:40.

23. Encuesta Nacional de Ocupación y Empleo. 2011. INEGI. (monografía en internet). México: INEGI,2011. (consultado 2012 agosto 11). Disponible en http://www.inegi.org.mx/sistemas/ tabuladosbasicos2/tabtema.aspx?s=estEc $=28822$.

24. Veryune JL, Tubert-Jeannin S, Dutheil C, Riordan PJ. Impact of new prostheses on the oral health related quality of life of edentulous patients. Gerodontology 2005;22:3-9.

\section{CORRESPONDENCIA}

Alejandra Moreno Altamirano.

Av. Universidad No. 3000

Ciudad Universitaria. CP. 04510

Departamento de Salud Pública, Edificio B, $6^{\circ}$ piso Facultad de Medicina, UNAM.

México DF

E-mail: gamoreno@servidor.unam.mx 